\title{
HAART and liver: A discussion of hepatic disease in those living with HIV
}

\author{
Curtis Cooper MD ${ }^{1}$, Paul C Adams MD FRCPC ${ }^{2}$, Editor-in-Chief
}

\begin{abstract}
C
urtis Cooper is an Associate Professor with the University of Ottawa, Infectious Diseases Consultant with the Ottawa Hospital Division of Infectious Diseases, and a researcher with the Ottawa Health Research Institute. His research interests are in the evaluation of the clinical, biochemical, virological, immunological, psychological and pharmacokinetic interactions among viral hepatitis, HIV and the medications used to treat these chronic infections.
\end{abstract}

PA: I was a resident in San Francisco in 1983 when AIDS seemed like a fatal epidemic of biblical proportions. At that time, health care personnel were very concerned about the transmission of the virus from patient to providers and clinical care on some occasions was less than ideal. Can you update us on the current status of this infectious disease?

CC: There have been dramatic improvements in the treatment of HIV since that time. The arrival of protease inhibitors in the mid 1990s represents a quantum change in HIV care. When combined with nucleoside analogues (eg, zidovudine, lamivudine, stavudine), complete and long-term HIV viral load suppression could be achieved for the first time. This resulted in immune function recovery, and a dramatic reduction in morbidity, hospitalization and death. Initially, these therapies were associated with significant difficulties including massive pill counts, multiple daily dosings, gastrointestinal intolerance, peripheral neuropathy, metabolic abnormality and body-shape changes, to name a few. Thankfully, combination antiretroviral therapy has advanced considerably since then. Now, when a newly diagnosed patient arrives at the clinic, we can often initiate a oncedaily regimen consisting of as little as one pill per day. We have multiple combination options that effectively suppress HIV virus indefinitely. The newer medications are much better tolerated, have fewer metabolic effects and appear to be free of the body habitus changes associated with earlier treatments. The upshot is if patients take their medications, they will maintain virological suppression, will not develop progressive HIV disease and can expect a life expectancy that approaches that of people without HIV. Of course, there are still concerns for those who are not adherent to therapy. Also, there are higher lymphoma and liver failure rates compared with the overall population, even in patients with well-controlled HIV.

I frequently see health care providers in my clinic following needlestick injuries. The fact that most patients on antiretroviral therapy have completely suppressed HIV viremia is a key factor when considering the risk of infection. I still recommend postexposure prophylaxis if someone has received a needlestick injury. However, if the source's HIV viral load is below detectable limits, I counsel them on the negligable risk of seroconversion. The risks of HIV infection while performing other medical procedures such as endoscopy or brochoscopy is infinitesimally small. These procedures are routinely performed on HIV patients by our colleagues.

PA: With the success of highly active antiretroviral therapy (HAART), liver disease has become a major cause of death in these patients. Can you comment on the treatment approach and how it may differ from a non-HIV patient?

CC: Liver disease has become a chief cause of mortality. This is true, even in those who have durable HIV viral load suppression and good immune recovery. This is primarily driven by hepatitis $\mathrm{C}$ virus (HCV) coinfection. Overall, approximately $20 \%$ to $25 \%$ of HIV patients are HCV coinfected. The prevalence of HIV-HCV coinfection depends on the setting in which you practice. In my own Ottawa clinic, more of my patients are former injection drug users and as a consequence the seroprevalence is $30 \%$ to $35 \%$. In the core of Vancouver, the coinfection prevalence is over $80 \%$. Just like in any liver practice, hepatitis B- and alcohol-induced liver disease are concerns as well.

There are some special considerations with HIV-HCV coinfection treatment. Ideally, I would first treat HCV. By doing this, one could avoid the combined side effects of both HCV and HIV antiviral therapy. This is less of a concern these days because antiretroviral therapies have much better side effect profiles. We do not use didanosine, which interacts with ribavirin to produce excess pancreatitis and liver toxicities. As well, we use less zidovudine, which in combination with ribavirin produces more severe anemia. There is some evidence

${ }^{1}$ Ottawa Hospital, General Campus, Ottawa, Ontario; ${ }^{2}$ University Hospital, London, Ontario

Correspondence: Dr Curtis Cooper, Division of Infectious Diseases, Ottawa Hospital, General Campus, 501 Smyth Road, Ottawa, Ontario

K1H 8L6. Telephone 613-737-8924, fax613-737-8164, e-mail ccooper@ottawahospital.on.ca

Received and accepted for publication March 31, 2008. 
that CD4 cell recovery is blunted by HIV-HCV coinfection following the initiation of antiretroviral therapy. I am not convinced that this is a direct HCV effect and in my judgment is more likely due to concurrent socioeconomic and substance abuse issues with which coinfected patients are more heavily burdened compared with HIV monoinfected patients. Nonetheless, some argue that by first successfully treating $\mathrm{HCV}$, you could subsequently maximize immune restoration achieved with HAART. There is a slightly greater risk of antiretroviral-related liver toxicities in those who are viral hepatitis coinfected. Some argue that it makes sense to first eliminate $\mathrm{HCV}$ infection and thereby minimize the risk of antiretroviral-related liver complications. I think each of these arguments has some merit. However, I typically do not first meet my coinfected patients when they have high CD4 counts and are in good health. More often, I first encounter my HIVHCV coinfected patients in the emergency department when they present with their initial opportunistic infection. In this context, HIV antiretroviral therapy is obviously required and the HCV infection is put on the back burner.

I typically focus on introducing antiretroviral therapy even when patients present with higher CD4 cell counts. The benefits in terms of HIV are clear. It is also evident that by managing HIV well, there are indirect benefits regarding the natural progression of viral hepatitis. It has been well established that patients on antiretroviral therapy progress less rapidly toward cirrhosis and die less frequently from liver-related complications when compared with those patients not on HAART. My Masters research demonstrated that HIV-HCV coinfected patients on long-term, virologically suppressive antiretroviral therapy have lower HCV RNA levels than they did before HAART was started. As you know, baseline HCV RNA levels are an important predictor of HCV therapy success. I think that this further supports the approach of first focusing on the HIV treatment and then, once this is established, turning your attention to HCV.

There is concern among patients and treating physicians that antiretroviral therapy, when dosed in a coinfected patient, will produce very high rates of hepatotoxicity. This is simply not the case. In fact, when we evaluated our own HIV-HCV coinfected population initiating a first round of antiretroviral therapy, we found that only $4 \%$ abandoned HIV treatment because of liver complications. In fact, our coinfected patients discontinued their therapies for the same reason that HIV monoinfected patients did - gastrointestinal intolerance and adherence issues. There is a lack of clarity regarding what is meant by 'hepatotoxicity'. An asymptomatic liver enzyme flare occurring in the first few months of antiretroviral therapy occurs in approximately $5 \%$ of coinfected patients. This is often associated with a spike in HCV RNA level. By six months, the liver enzymes have usually returned to normal. In fact, the liver enzymes are often lower than that of baseline at one year. What I am far more concerned with is the occurrence of clinically relevant liver toxicity where the patient develops jaundice or signs of decompensation. This occurs in $1 \%$ to $2 \%$ of coinfected patients initiating antiretroviral therapy. In the past, drugs such as stavudine, which is not used much anymore, and high-dose ritonavir were implicated as being particularly liver unfriendly. We currently use low-dose ritonavir, which possesses no greater potential for liver complications than other standard of care medications. I don't mean to minimize my concern related to antiretroviral-related liver toxicity. I will ask coinfected patients to return to clinic two weeks after the initiation of therapy to evaluate them and ensure that their liver function tests and liver enzymes are within acceptable limits. However, antiretroviral therapy should not be delayed or inferior medications used because of unfounded fear related to liver toxicity.

My approach to HCV management in a HIV-HCV coinfected patient is very similar to someone who is HCV monoinfected. Although there is a greater burden of liver fibrosis overall and HCV progression is more rapid in coinfected patients, this is not universal. As in HCV monoinfection, I often pursue a biopsy to help establish the extent of fibrosis within the parenchyma of the liver. If an individual has very little fibrosis after years of infection, I will generally not recommend therapy, irrespective of their HIV status. The same issues (eg, likelihood of adherence, substance abuse, mental health, comorbid disease, socioeconomic status) that go into assessing the potential efficacy and safety of HCV therapy in HCV monoinfected patients are considered for patients with HIV. Several large clinical trials in HIV-HCV coinfected patients suggest that the safety and tolerance of HCV treatment is comparable with those with HCV monoinfection. Unfortunately, the likelihood of sustained virological response is lower in coinfection (genotype 1:30\% to $40 \%$, genotype $2 / 3$ : $50 \%$ to $60 \%$ ). Nonetheless, we have had many successes in our clinic. Given the fact that these patients now have much longer life expectancies, it makes sense to evaluate HIV patients for HCV therapy.

The approach to hepatitis B virus (HBV) is somewhat different. Medications including tenofovir, lamivudine and emtricitibine (a close cousin of lamivudine) have antiviral activity against both HIV and HBV. Therefore, when HIV therapy is started, it makes sense to include both tenofovir and lamivudine/FTC in the regimen. This approach achieves better HBV DNA suppression, produces greater liver enzyme normalization and slows the evolution of HBV drug resistance. One must avoid initiating one of these oral medications (or entecavir) as HBV monotherapy in a HIV-HBV coinfected patient. This will produce HIV drug resistance and as a consequence, you will lose medication options for the future. Given the fact that HBV liver disease progresses more rapidly in most people with HIV, many, including myself, argue that antiretroviral therapy should be initiated sooner (ie, at a higher CD4 count) in this population. In a HIV-HBV coinfected patient who truly requires only HBV treatment, then I think that interferon or telbivudine therapy are good options.

PA: Why do HIV patients have greater access to these medications than HBV patients?

CC: The inability to treat HBV monoinfection appropriately is very frustrating given limited medication coverage by provincial formularies. Tenofovir and lamivudine are covered under HIV medication reimbursement programs in most provinces. This facilitates access for HBV treatment in a coinfected patient. However, when I want to prescribe an alternate medication such as telbivudine or interferon, my HIV patient is in the same boat as everyone else.

There are barriers to delivery of HIV care in Canada. Like many medical conditions, it often takes an excessively long time for provincial formularies to assume the cost of newer antiretrovirals. Some provinces will cover a portion of HIV medication cost but this still leaves a patient responsible for thousands of dollars per year. This creates a financial crisis for 
someone living on the poverty line. Many of our foreign-born patients run into gaps in coverage that make remaining on uninterrupted antiretroviral therapy problematic.

I think one of the reasons why HIV patients have historically had greater success in obtaining the medications they require is that many are well-educated, well-off, well-organized vocal lobbyists with the time to invest in their own advocacy and the political clout necessary to successfully defend their rights. This is not the case for many of our HBV- and HCVinfected patients. Many lack advanced education, speak little English or French (ie, immigrants), come from depressed socioeconomic regions and are not politically connected. This explains some of the discrepancies in health care services provided to HIV patients compared with those who have viral hepatitis.

PA: What have we learned from HIV therapy that could be applied to the treatment of HBV?

CC: One important lesson learned from HIV therapy is the importance of adherence. Although it is not the most glamorous thing you can do in your medical practice, spending the extra time to educate a patient on the need for high levels of adherence will pay dividends. This is a focus of research and patient intervention in our HIV clinic. As a consequence, we have a lower burden of multidrug-resistant virus-infected patients.

PA: What do we know about combination versus sequential therapy in regard to drug resistance?

CC: Current HBV management is similar to that of HIV in the early 1990s. At that time, we had several nucleoside analogues including zidovudine, lamivudine, didanosine and stavudine. By themselves or in dual combination, they produced some degree of virological suppression but, because the viral loads could not be completely suppressed, drug resistance eventually developed and the patient eventually succumbed to AIDS-related disease. It was only once the protease inhibitors and non-nucleoside reverse transcriptase inhibitors (eg, efavirenz, nevirapine) became available that we had the 'guns' to achieve full suppression of the HIV. It is the same with current HBV therapy. Whether we use one, two or more of the currently available nucleoside analogues, they will not fully suppress viremia if it is very high at baseline. Ongoing viral replication in the presence of sufficient antiviral drug plasma levels will almost assuredly result in resistance given enough time. There is evidence that combination therapy will delay the development of resistance; however, as with HIV treatment in the early 1990s, resistance will eventually happen. What are needed are other HBV antiviral classes but there are no apparent candidates at the moment.

PA: Is the complexity of treatment for HIV and hepatitis becoming so complicated that an average physician should opt for referral to special treatment centres?

CC: With HIV and viral hepatitis, there is great potential to cause considerable harm if the medications are used incorrectly because drug resistance, treatment failure and further progression of the disease state will result. I think it is in the patient's best interest to be managed by a physician who has experience with HCV, HBV and/or HIV, knows the medications well, has the required support staff and is prepared to spend the time required to help the patient through the challenging therapies offered. It seems that HCV treatment is going to get more complicated as protease inhibitors and nucleoside analogues become available within the next few years. Interpreting viral load changes over time, resistance profiles and pharmacokinetic interactions can be complex.

PA: Can you update us on your perspectives on liver transplantation for HIV patients in Canada?

CC: In the old days, when we lacked good HIV therapy, it did not make any sense to consider liver transplantation. The situation is now different. Concerns that post-transplant antirejection medications would result in immunological collapse have been dispelled. It has been suggested that the polypharmacy required to manage both HIV and a liver transplant should preclude transplantation. However, the pill burden for an average HIV patient is now quite manageable. Furthermore, we routinely transplant patients on multiple medications for hypertension, lipid abnormalities and other chronic diseases. There are significant interactions between medications (eg, tacrolimus and HIV protease inhibitors) but catastrophe can be avoided assuming that a pharmacist who is well-versed in the use of these medications is part of the transplant team and there is good communication between the liver transplant and HIV care teams. There have been concerns raised about longterm malignancy risk (eg, lymphoma, human papilloma virusrelated cancers) in a transplanted HIV patient. To date, this fear has not come to fruition. As a fellow health care provider, I appreciate concerns related to needlestick injury risk and appreciate that a liver transplant procedure is a technically challenging endeavour. However, most HIV patients receiving a transplant will be on antiretroviral therapy and will have no detectable virus in their blood. The risk of acquiring HIV infection from a patient like this is negligible. Frankly, I am far more concerned about the risk of HCV infection following a needlestick injury given the fact that the typical hepatitis C viral load is quite high. Despite this, we routinely transplant individuals with $\mathrm{HCV}$ monoinfection. When you review the literature from transplant centres in the United States, France, Spain, Italy and England, the three to five year survival is comparable with individuals without HIV. I am not suggesting that HIV-infected patients receive preferential consideration. You would never want to see a liver go to a patient where it is doomed to failure. To this end, the same strict criteria pertaining to mental health, substance abuse, concurrent illnesses and likelihood of medication adherence needs to be rigorously applied to all patients, including those with HIV. Having said this, there is a clear need among HIV patients and a body of work that suggests that this can be safely and effectively accomplished in those living with HIV. They should be given a fair shake like everyone else. 


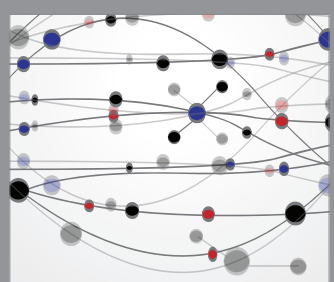

The Scientific World Journal
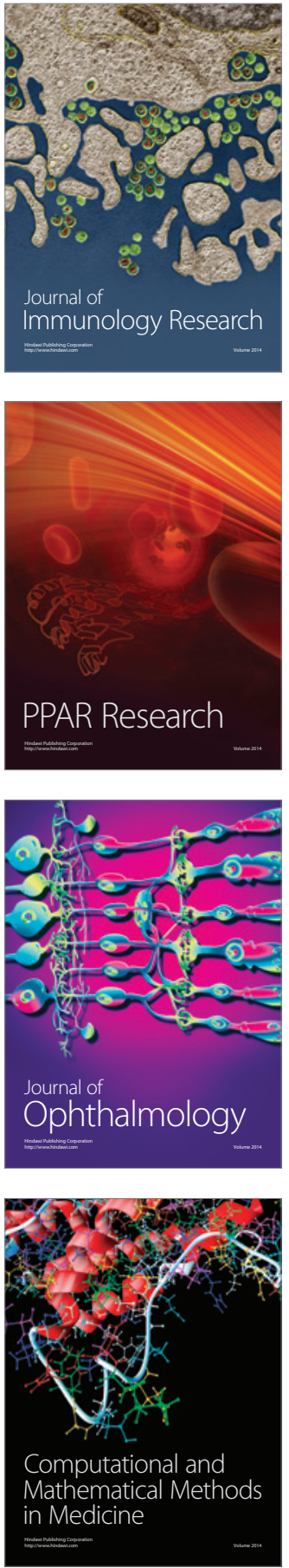

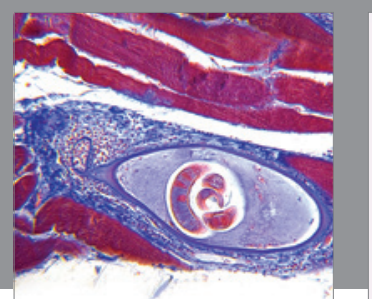

Gastroenterology Research and Practice

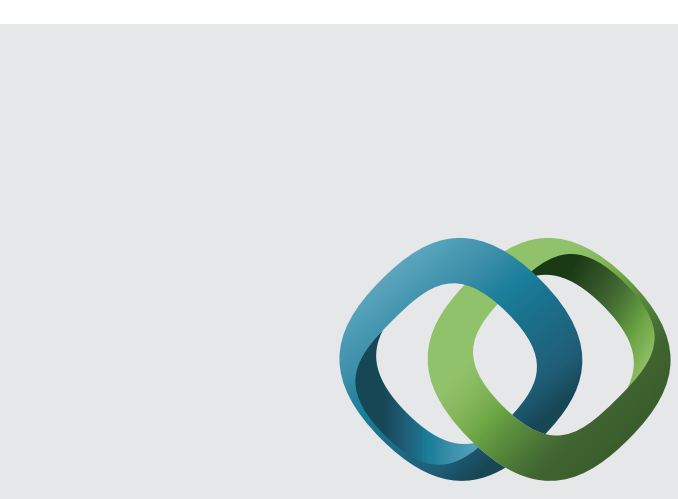

\section{Hindawi}

Submit your manuscripts at

http://www.hindawi.com
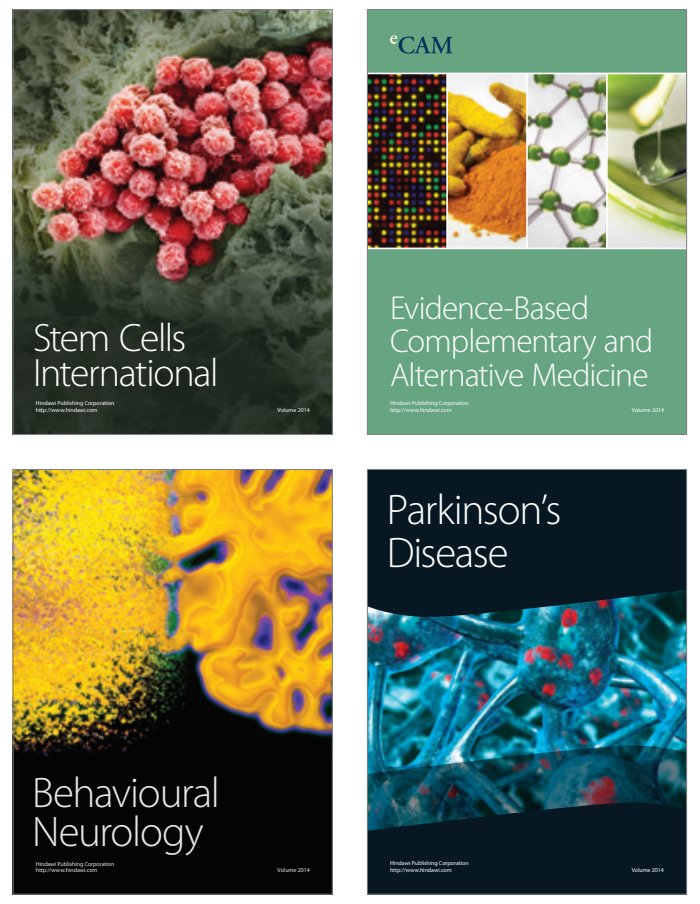
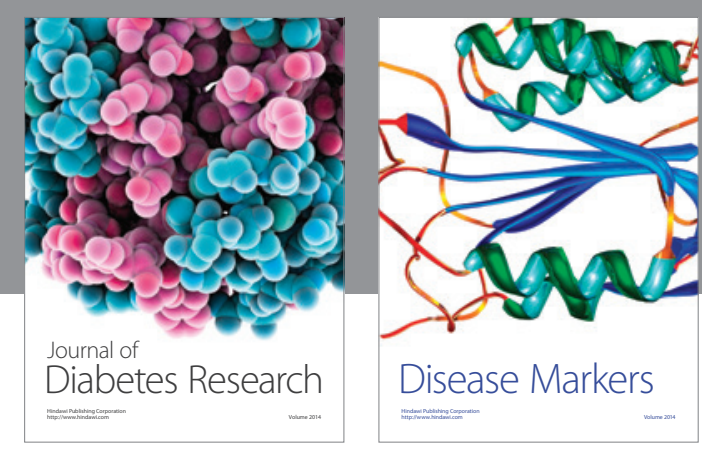

Disease Markers
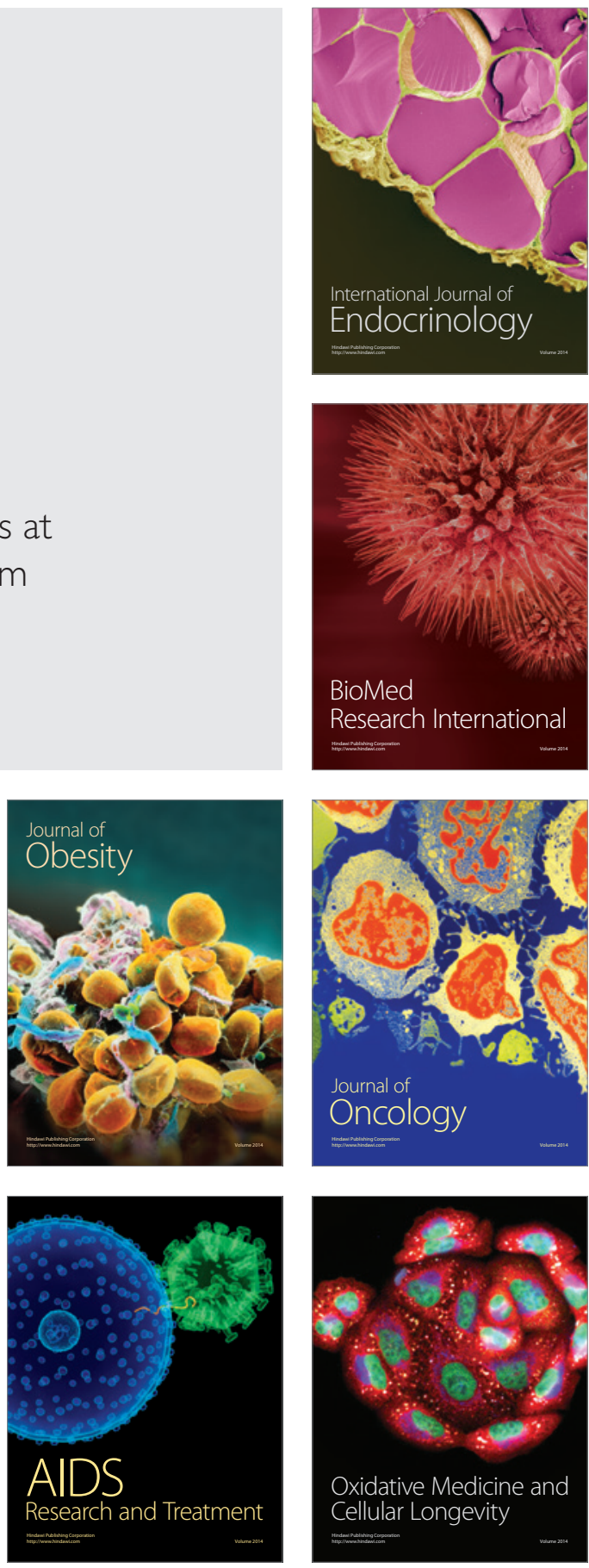